\title{
Integration of features in depictions as a function of size
}

\author{
DARRELL L. BUTLER \\ Ball State University, Muncie, Indiana \\ and \\ ANN M. KRING \\ State University of New York, Stonybrook, New York
}

\begin{abstract}
Prinzmetal (1981) has argued that the visual system does not integrate features on the basis of their proximity to one another; rather, the visual system is likely to integrate features from the same perceptual group regardless of their proximity. The present paper examines this hypothesis by studying the effect, on judgments of $90^{\circ}$ corners, of several geometric characteristics that can affect perceptual organization and can be spatially remote (parallel lines, enclosure, and regularity of line length). Results suggest that both parallel lines and enclosure can affect $90^{\circ}$ corner judgments even when the parallel lines and enclosure lines are spatially remote from the corner judged. Regularity of line length was found to affect judgments only of small stimuli. The results partially support Prinzmetal's hypothesis. Emergent features provide a better explanation of obtained results.
\end{abstract}

Prinzmetal (1981) has argued that the visual system is likely to integrate features from the same perceptual group or object regardless of proximity. According to Prinzmetal, the likelihood of integration is thus determined by perceptual organization, that is, similarity, goodness of form, and so forth.

The purpose of the present experiments was to examine several geometric characteristics that can theoretically affect perceptual organization and can theoretically be spatially remote: parallel lines, enclosure, and regularity of line length. The goal was to provide initial exploration of the integration of parallel lines, enclosure, and regularity of line length with small (i.e., local) features in drawings as a function of proximity between the geometric features and the local features.

Many psychologists believe that parallelism is a powerful cue in object perception (e.g., Attneave, 1982; Perkins \& Cooper, 1980; Rock, 1983, p. 155). In Attneave's dipole theory of visual perception (his recent version of Gestalt reasoning), regularities, including parallelism, are used to determine percepts. Perkins and Cooper assimilate parallelism into the Gestalt law of simplicity in their hypothesis that the visual system seeks out simple, geometric regularities, including parallelism, and uses them to resolve projective ambiguities in form perception. Rock argues that spatial relationships such as parallelism must be contained in the perceptual descriptions of form. He suggests that parallelism in pictures may even be accepted as representing parallelism in objects even though the

This research was supported by a grant from Ball State University awarded to the first author. Requests for reprints should be sent to Darrell L. Butler, Department of Psychological Sciences, Ball State University, Muncie, IN 47306. projection is incorrect. These researchers have not empirically demonstrated their hypotheses about parallelism and, more importantly, none of them have examined the question of whether these effects vary with stimulus size.

Enclosure is another stimulus characteristic that is not necessarily detectable in a single fixation of a small region of the visual field. Mathematically, enclosure can be defined as a contour described by a periodic function in parametric equations. However, some closure or "filling in of the gaps" occurs in perceptual judgments of enclosure. Thus, perceptual closure is difficult to describe mathematically. Gibson (1982) defines enclosure as follows: "An object is only a surface that stands out from the rest of the surface layout, the ground, because it is bounded by an occluding edge" (p. 283). An occluding edge refers to the outermost edge of a three-dimensional object or its depiction. Several researchers (e.g., Barrow \& Tennebaum, 1981; Koffka, 1935; Marr, 1982; Rock, 1983) have agreed that identifying the occluding edge of an object is an important stage in object perception, and some have argued that the enclosed region is an emergent feature (Pomerantz, 1986; Treisman \& Paterson, 1984).

The influence of regularity of line length, another possible nonlocal aspect of a stimulus, has been shown to have several perceptual consequences. Attneave and Frost (1969) have shown that regularity of line length can affect the tridimensionality of depicted objects. Attneave (1982) has incorporated regularity into his recent theory, but provides no additional evidence for its inclusion. Recent studies using Leeuwenberg's perceptual coding theory (e.g., Buffart, Leeuwenberg, \& Restle, 1983; Butler, 1982) have supported the basic supposition that regularity of line length can be important in perceptual tasks. 
The aim of the present experiments was to determine whether enclosure, parallel lines, and regularity of line length are integrated with other features (that is, affect perceptual judgment of other features) across a wide variety of sizes of stimuli. Experiment 1 established a method for studying perceptual integration. The basic logic was to show that the perceptual interpretation of a feature is affected by another feature in the stimulus, that is, that selective attention fails. Experiment 2 investigated the question of whether the integration among features varies with stimulus size.

\section{EXPERIMENT 1}

The purposes of this experiment were to establish a method for studying feature integration and to provide a basis for analyzing the effect of parallel lines, enclosure, and regularity (equality of line length). The experiment examined the effect of these variables on the perception of $90^{\circ}$ corners in line drawings. Butler (1984), Perkins (1972), and Shepard (1981) have shown that there is a bias toward seeing corners in line drawings as $90^{\circ}$ corners when such interpretations are consistent with projective geometry. Perkins (1972) and Shepard (1981) found that individuals were fairly accurate in identifying depicted $90^{\circ}$ corners. However, Butler (1984) has shown that the strength of the $90^{\circ}$ corner bias is influenced by context.

The present experiment used the context sensitivity of the $90^{\circ}$ corner bias to explore the effects of enclosure, parallelism, and regularity on perception of shapes in line drawings.

\section{Method}

Subjects. The subjects were 26 undergraduates in the research participant pool at Ball State University. Subjects with uncorrected eye problems (self-report) were excluded.

Stimuli. The stimuli were 16 line drawings created on a highresolution, 22-cm black-and-white monitor connected to an Apple II+ computer. The center of each stimulus was a three-line intersection defined by solid lines about $1 \mathrm{~mm}$ wide. The angles in the intersection were chosen such that the three-dimensional interpretations either could be a $90^{\circ}$ corner $\left(120^{\circ}, 120^{\circ}\right.$, and $\left.120^{\circ}\right)$ or could not be a $90^{\circ}$ corner $\left(60^{\circ}, 160^{\circ}\right.$, and $\left.160^{\circ}\right)$ by parallel projection in which the intersection was in the center of the visual field. The length of the lines in the three-line intersections varied. Eight stimuli (the regular drawings) contained three equal line lengths $(27 \mathrm{~mm}$, or $1.3^{\circ}$ of visual angle in this experiment). The other eight drawings (the irregular drawings) contained lines unequal in length (20, 27 , and $33 \mathrm{~mm}$ ). Other lines and angles in the stimuli were also varied. Two lines were appended to the end of each of the lines that formed the three-line intersection. These appended lines created either an open or an enclosed figure and were either parallel or nonparallel to the lines in the center intersection. These context lines were drawn in dotted lines approximately $1 \mathrm{~mm}$ wide. Distance between the appended lines depended on whether the stimuli could have a $90^{\circ}$ corner and on regularity. In regular stimuli that could have a $90^{\circ}$ corner, appended lines were attached to line endpoints that were $2.5^{\circ}$ of visual angle apart. In regular stimuli that could not have a $90^{\circ}$ corner, the appended lines were attached to line endpoints that were $1.5^{\circ}$ or more of visual angle apart. Examples of the stimuli are shown in Figure 1.

These four variables, center intersection angles (can be $90^{\circ}$ corner/cannot be $90^{\circ}$ corner), center intersection lines (equal

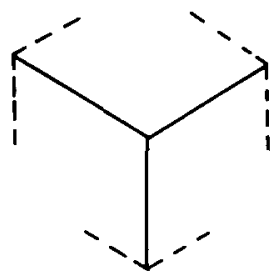

(a)

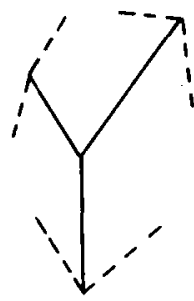

(c)

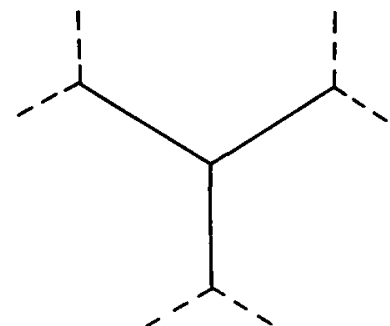

(b)
Figure 1. Examples of stimuli used in Experiment 1. Examples a and $b$ can be $90^{\circ}$ corners, are regular, and contain parallel lines. They differ in that Example $a$ is enclosed and Example $b$ is not enclosed. Example c cannot be a $90^{\circ}$ corner, is irregular, does not contain parallet lines, and is enclosed. Example $d$ is the same as Erample c except that it is not enclosed.

length/unequal length), enclosure (open figure/enclosed figure), and paralleiness of appended lines (parallel/not parallel), were completely crossed to create 16 stimuli.

Procedure. The subjects were run individually. Each subject sat in a chair approximately $122 \mathrm{~cm}$ from the CRT. A 40-W red light dimly illuminated the written instructions, and a low-wattage white bulb indirectly illuminated the walls behind the subject. The subject read the instructions while simultaneously listening to a tape recording of the instructions. All subjects received the same set of instructions.

To learn how to use the buttons connected to the computer, the subjects first went through a training procedure. Pilot studies had strongly indicated that without this training naive subjects produced very unreliable reaction times. The task involved making simple discriminations between line drawings of triangles and squares presented one at a time on the CRT. The subjects responded by pressing one of two buttons: the right-side button for a square and the left-side button for a triangle. The subjects were instructed to respond as quickly as possible without making mistakes. Altogether, 50 triangles and $\mathbf{5 0}$ squares were presented. Reaction time (RT) feedback was given on each trial, and mean RT and percent correct feedback was provided at the end of training.

Next, the subjects were given instructions for the experiment. They were told that they were to make judgments about the center corner of drawings depicting three-dimensional things. Specifically, they were to decide if the designated corner could be a $90^{\circ}$ corner. A $90^{\circ}$ corner was defined as a corner in which each surface contained a $90^{\circ}$ angle and all surfaces were perpendicular to each other. Four examples of depicted corners (the corners to be used in the experiment, but not shown with any context lines) were shown one at a time on the CRT along with a statement indicating whether the stimulus could or could not be a $90^{\circ}$ corner. The subjects were able to view the examples as many times as they wished and for as long as they wished. During the experiment, the subjects were 
to respond as quickly as possible without making mistakes in using the buttons. The right-side button was for a "yes" ("it can be a $90^{\circ}$ corner") response, and the left-side button was for a "no" ("it cannot be a $90^{\circ}$ corner") response. The subjects were also instructed to sit in an upright position and not to move around much during the experiment.

Each stimulus was displayed on the CRT until the subject pressed a button. Then the CRT was blanked for about 5 sec, after which a small " +" was printed in the center of the CRT (where the center of the intersection of the next stimulus would be presented) and a message appeared on the CRT telling the subject to press either button to begin the next trial. The next stimulus was presented $500 \mathrm{msec}$ after the buttonpress. The stimuli were presented in six blocks of 16 trials for a total of 96 trials. Order of stimuli was randomized independently for each subject, for each block.

\section{Results}

All three cues that can be spatially remote (regularity, enclosure, and parallel lines) had an effect on RT and accuracy. The interactions were important, as described below.

Because accuracy of judgments was near chance (40 errors in 96 judgments) for 1 subject, the results of that subject were not included in the analyses. For the other 25 subjects, accuracy and RTs were analyzed separately using analysis of variance. For accuracy, the dependent variable was the percent correct for each stimulus for each subject averaged across repetitions. RTs were analyzed using the median RT for each subject across correct judgments of each stimulus.

Parallel lines did not substantially affect RTs for open figures, but parallel lines had a strong influence on enclosed figures. The three-way interaction between parallelness, enclosure, and comer was significant $[F(1,24)=4.66, p<.05]$. The means involved in this interaction are shown in Figure 2a. The mean standard (a)

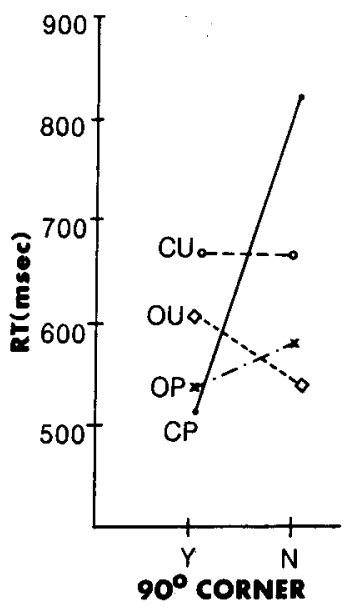

(b)

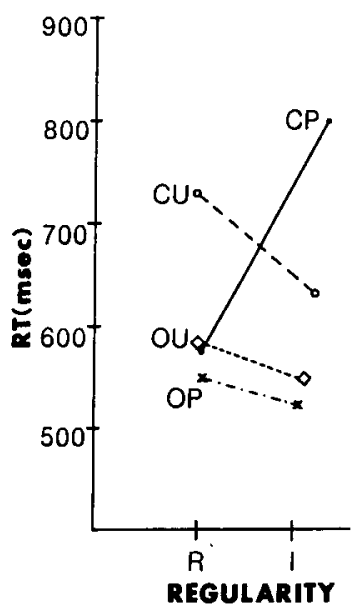

Figure 2. Mean RTs for judgments in Experiment 1. (a) The interaction between enclosure ( $O=$ open, $C=$ closed), parallelness ( $P$ $=$ parallel, $U=$ nonparalle), and corner $\left(Y=90^{\circ}\right.$ corner, $\mathbf{N}=$ not a $90^{\circ}$ corner). (b) The interaction between enclosure, parallelness, and regularity ( $R=$ regular, $I=$ irregular). (a)

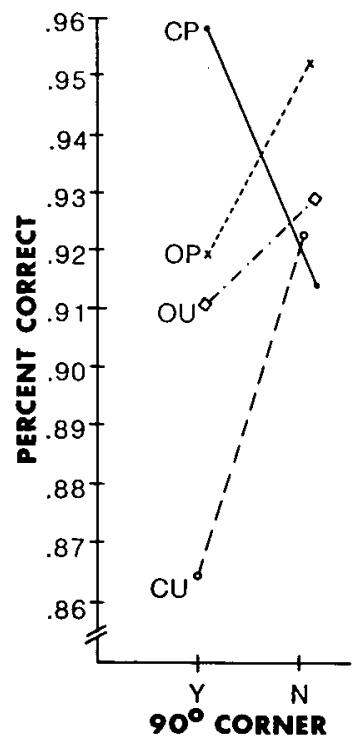

(b)

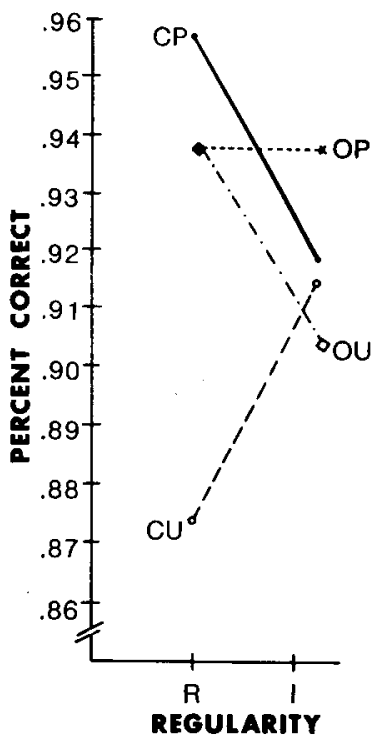

Figure 3. Percent correct for judgments in Experiment 1. (a) The interaction between enclosure, parallelness, and corner. (b) The interaction between enclosure, parallelness, and regularity. (See Figure 2 for key.)

error of these means is 64 msec. As can be seen in the figure, means for the eight open figures do not differ greatly from one another. The means among the eight enclosed figures do vary substantially. Responses to enclosed figures containing parallel lines and a $90^{\circ}$ corner (i.e., a rectangular solid) were more rapid than responses to any other enclosed figure. Responses to enclosed figures containing parallel lines and a non- $90^{\circ}$ corner were slower than responses to any other enclosed figure.

The three-way interaction between parallel lines, enclosure, and regularity was also significant $[F(1,24)=$ $8.26, p<.01]$. The means involved in this interaction are shown in Figure $2 b$. The means of the open stimuli do not differ substantially. Responses were more rapid to enclosed figures with regular, parallel lines than to other enclosed figures. Responses to enclosed figures with irregular, parallel lines were slower than responses to other enclosed figures. For enclosed figures with nonparallel lines, the opposite effect of regularity was found; responses were more rapid to irregular figures than to regular figures.

A number of other main effects and two-way interactions were significant. However, their interpretation is superseded by the three-way interactions described above. The four-way interaction was not significant.

Time-accuracy tradeoffs were not responsible for the major results indicated above. A summary of response accuracy is given in Figures $3 a$ and $3 b$. The three-way interaction between corner, enclosure, and parallel lines was significant $[F(1,24)=17.31, p<.001]$, but the three-way interaction between regularity, enclosure, and 
parallel lines was not significant at the .05 level $[F(1,24)$ $=3.49, p=.07]$. Overall, the data are quite consistent with the RT data; high accuracy rates generally correspond to faster RTs. Note, however, that accuracy data indicate that the interaction between enclosure, corner, and parallel lines may be slightly different from that indicated by RTs. Accuracy of enclosed figures without parallel lines varied as a function of corner type, but these figures did not lead to substantially different RTs.

The robustness of the above results was examined with two further analyses. First, accuracy data were reanalyzed using only the 19 subjects who had error rates below $10 \%$. The same qualitative patterns showed in the analysis, although the means and variances were somewhat smaller. Both three-way interactions described above were significant at about the .01 level. Robustness was also examined by computing the median RTs across subjects. This was done because some subjects had such high error rates that their median RT was based on only one judgment. The results quite closely resemble the means shown in Figures $2 \mathrm{a}$ and $2 \mathrm{~b}$. Two differences between medians and means were found. Overall, medians were about $170 \mathrm{msec}$ faster than the means. Also, with the accuracy data, the threeway interaction between parallelness, enclosure, and corner is more consistent (visually) when medians are considered than when means are considered.

The subjects were interviewed at the end of the experiment. Surprisingly, they reported that they were able to focus on the intersection at the center of the CRT and ignore the other lines in the display. None of the subjects believed that his or her judgments were affected by the context lines.

\section{Discussion}

This experiment illustrates a method for examining a feature integration, and suggests that regularity, parallelness, and enclosure all affect judgments of $90^{\circ}$ comers. Consistent with arguments made by Prinzmetal (1981), this experiment suggests that integration depends on features' being in the same "object." For these experiments, an object would probably be best defined as either an enclosed region in the visual field or an apparent 3-D object, not just a set of connected lines.

For enclosed figures, parallel lines facilitated seeing $90^{\circ}$ corners and inhibited seeing a non- $90^{\circ}$ corner. The effect of enclosure found in this experiment could be due to differences in the sizes of the stimuli. In this experiment, enclosed figures were generally smaller than open figures. Perhaps enclosed figures only produced context effects because they were smaller than open figures. This hypothesis was considered in Experiment 2.

The finding that parallel lines affected judgments of closed figures could be due in part to a response facilitation resulting from familiarity with rectangular boxes. However, familiarity with rectangular boxes is not a sufficient explanation for all of the results. Both accuracy and RT data suggest that nonparallelness leads to different judgments in open and closed figures. This result is difficult to explain by familiarity with rectangularity.

\section{EXPERIMENT 2}

Experiment 1 indicated that several cues could affect the $90^{\circ}$ corner bias. However, the stimuli were relatively small, and closed stimuli were somewhat smaller than open stimuli. In Experiment 2, overall stimulus sizes were varied over a wider range so that the effect of size could be determined.

\section{Method}

Subjects. The subjects were 29 undergraduates in the research participant pool at Ball State University. None of the subjects had participated in Experiment 1. None of them had uncorrected eye problems, according to self-report.

Stimuli. Two sets of stimuli were created from those used in Experiment 1 . The two sets differed in size of the stimulus. One set was 1.25 times the size of those used in Experiment 1, and the other set was .31 times the size of those used in Experiment 1. However, the subject-CRT distance was changed from that used in Experiment 1 , such that the large stimuli in this experiment were twice the visual angle of those used in Experiment 1 and the small stimuli in this experiment were half the visual angle of those used in Experiment 1. Also, in this experiment, the context lines were drawn in solid lines rather than in dotted lines as had been done in Experiment 1. In addition, enclosed figures were completely enclosed. In other respects, the stimuli were the same in this experiment as in Experiment 1.

Procedure. The procedure was the same as that in Experiment 1 except for the following changes. In this experiment, subjects sat in a chair approximately $76 \mathrm{~cm}$ from the CRT (in Experiment 1 they were $122 \mathrm{~cm}$ away). The 32 stimuli were presented throe times, in blocks, for a total of 96 trials. Order of stimuli was independently randomized for each block, for each subject.

\section{Results}

The results basically replicated the findings in Experiment 1. In addition, there was no effect of size on the interaction among parallel lines, enclosure, and corner, but size affected the interaction between regularity, enclosure, and parallel lines.

The results of one subject with 34 errors were not included in the analyses. Although error rates for other subjects were lower, they were high for particular conditions in the experiment. For this reason, RTs could not be analyzed as they had been in Experiment 1. Overall, the mean RT was $609 \mathrm{msec}$ and the median was $470 \mathrm{msec}$. These measures are similar to those obtained in Experiment 1. Accuracy of responses was analyzed as in Experiment 1.

As found in Experiment 1, parallel lines did not substantially affect accuracy for open figures, but did affect accuracy of enclosed figures. There was a significant interaction between parallel lines, enclosure, and corner $[F(1,27)=25.97, p<.001]$. The means are shown in Figure 4. Differences among the means of enclosed figures are much larger than the differences among the means of open figures. As found in Experiment 1, accuracy was highest for an enclosed figure containing parallel lines and a $90^{\circ}$ corner. Also, accuracy was lowest for an enclosed figure containing nonparallel lines and a non- $90^{\circ}$ corner.

The interaction between regularity, enclosure, and parallel lines varied substantially with size. The interaction 
between size, regularity, enclosure, and parallel lines was significant $[F(1,27)=5.09, p<.05]$. The means are shown in Figure 5. As shown in Figure 5, the interaction for smaller stimuli is similar to that found in Experiment 1 , although there are some differences. The differences among the means of enclosed figures is much larger here than in Experiment 1, while the differences among the means of open figures is about the same in the two experiments. For the larger stimuli, the interaction between the means is smaller than that found in Experiment 1 . For the larger stimuli, the errors for all stimuli were fewer than $10 \%$.

Although a number of other main effects and interactions were significant, their interpretation is superseded by the interactions described above.

As in Experiment 1, subjects reported that they were able to ignore the context lines. However, they also reported that the smaller stimuli were harder to judge than were the larger stimuli.

\section{GENERAL DISCUSSION}

These experiments demonstrate a reliable integration of features in the perception of depicted objects. Judgments of $90^{\circ}$ corners were affected by parallel lines in enclosed objects, even when the parallel lines were spatially distant from the judged corner. This finding is consistent with Prinzmetal's (1981) hypothesis that the visual system is likely to integrate features from the same perceptual group regardless of proximity. However, these experiments do not entirely support his hypothesis that

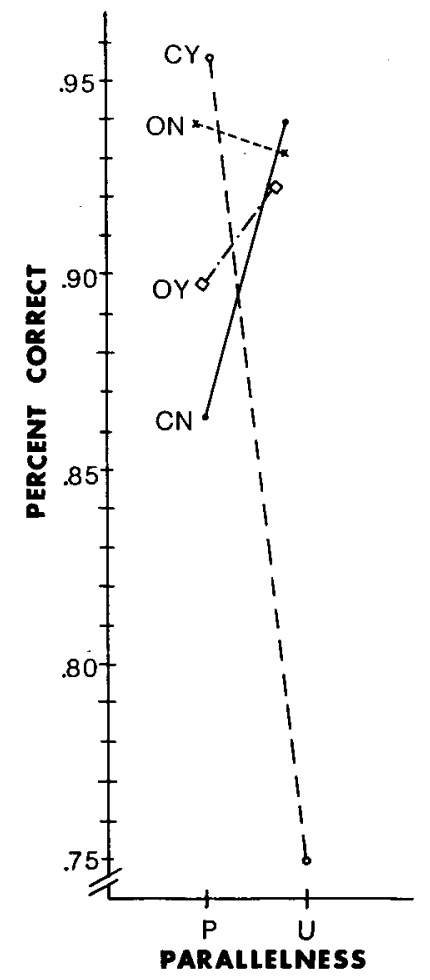

Figure 4. Percent correct for judgments in Experiment 2 as a function of enclosure, parallelness, and corner. (See Figure 2 for key.)
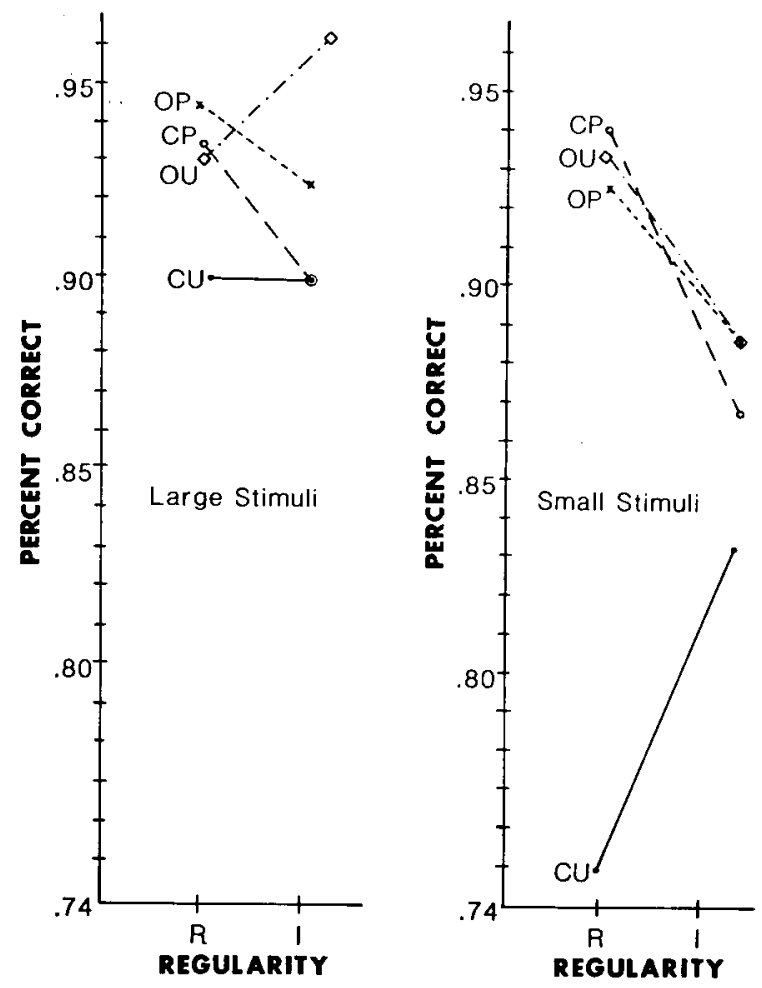

Figure 5. Percent correct for judgments in Experiment 2 as a function of size, enclosure, parallelness, and regularity. (See Figure 2 for key.)

integration is thus determined by perceptual organization. Regularity of line length, a factor many researchers have described as important in perceptual organization (e.g., Buffart et al., 1983), was not found to be an important factor in perceptual integration with large stimuli, even though it is a factor with small stimuli.

\section{Eye Movements}

A number of researchers have argued that integration occurs as a function of eye movements (e.g., Hochberg, 1981,1982 ). This is an unlikely explanation of the present results. Although stimuli remained in view until responses were made, no effect of size on mean or median RT was found across experiments or within experiments. Thus, any eye movements that occurred were equivalent across sizes of stimuli. We think, for two reasons, that it is unlikely that large eye movements occurred across conditions. First, subjects reported that they had attended to the center angle and had attempted to ignore the context. Second, the overall RTs were quite fast; the median RT was $\mathbf{4 7 0}$ msec in Experiment 2. For relatively unpracticed subjects, this is very fast responding, faster than one could expect if large eye movements were regularly occurring.

\section{Emergent Features}

Results suggest that parallelism and enclosure are emergent features arising from information integrated from peripheral and foveal vision. Previous research has suggested that peripheral vision contributes to sequential posi- 
tion of the eyes in reading (Rayner, 1978) and in object perception (Hochberg, 1981). The implication of the present experiment is that the periphery is involved not only in identifying location, but also in processing higher order features such as parallelism and enclosure.

Recent research by Treisman and Paterson (1984) and Treisman and Souther (1985) is consistent with these findings about closure. They found that closure, but not connectedness, appears to be a functional feature in visual perception. That is, closure seems to be an important emergent feature that is extracted early in perception and can lead to illusory conjunctions. Caelli, Julesz, and Gilbert (1978) proposed that closure is a perceptual primitive in texture discrimination. However, more recently, Julesz (1981) has discounted the role of closure and has, instead, emphasized the role of line terminators. In Julesz's approach, closure is simply the lack of line terminators. The results of the present research do not support this approach. In Experiment 1, closure was implied, but all figures contained the same number of line terminators. Nevertheless, an effect of enclosure was still obtained. Thus, enclosure is not simply the lack of line terminators.

To our knowledge, no previous research has demonstrated the functional importance of parallelness. Research involving texture discrimination, illusory conjunctions, or other tasks will be required to establish the reliability of parallelness as an emergent feature.

It is interesting to note that the effects of enclosure and parallelism in the present experiment can be thought of as a demonstration of failure of selective attention to angles of corners. In this regard, the experiment is quite unique. Unlike previous researchers (see summary by Pomerantz, 1986), we found no effect of size on the degree of this failure.

This finding is inconsistent with the results of several experiments that have shown that processing is different in fields larger than $2^{\circ}-3^{\circ}$ of visual angle. For example, von Gruenan, Wiggin, and Reed (1984) found that adaptation to a particular perspective orientation of the Necker cube is restricted to about $3^{\circ}$ of visual angle. Also, Pomerantz and Schwaitzberg (1975) reported that selectiveattention and divided-attention tasks were dependent on the size of the visual angle that separated the features being processed. They argue that separations larger than $2^{\circ}-3^{\circ}$ of visual angle lead to processing that is qualitatively different from that used with smaller separations. We found that regularity of line length acts very much as predicted by these researchers. However, enclosure and parallelism do not.

\section{REFERENCES}

Atrneave, F. (1982). Pragnanz and soap bubble systems: A theoretical exploration. In J. Beck (Ed.), Organization and representation in perception. Hillsdale, NJ: Erlbaum.
AtTneave, F., Frost, R. (1969). The determination of perceived tridimensional orientation by minimum criteria. Perception \& Psychophysics, 6, 391-396.

Barrow, H. G., * Tennebaum, J. M. (1981). Interpreting line drawings as three dimensional surfaces. Artificial Intelligence, 17, 75-116.

Buffart, H., LeEuWenberg, E., \& Restle, F. (1983). Analysis of ambiguity in visual pattern completion. Journal of Experimental Psychology: Human Perception \& Performance, 9, 980-1000.

BUTLER, D. L. (1982). Predicting the perception of three-dimensional objects from the geometrical information in drawings. Journal of Experimental Psychology: Human Perception \& Performance, 8, 674-692.

BUTLER, D. L. (1984, November). 3-D judgment of angles as a function of pictorial context. Paper presented at the 14th Annual Meeting of the Psychonomic Society, San Antonio, TX.

Caelu, T. M., Julesz, B., * Gilbert, E. N. (1978). On perceptual analyzers underlying visual texture discrimination: Part II. Biological Cybernetics, 29, 201-214.

GrBson, J. J. (1982). What is involved in surface perception? In J. Beck (Ed.), Organization and representation in perception. Hillsdale, $\mathrm{NJ}$ : Erlbaum.

HochBerg, J. (1981). Levels of perceptual organization. In M. Kubovy \& J. R. Pomerantz (Eds.), Perceptual organization. Hillsdale, NJ: Erlbaum.

HochBERG, J. (1982). How big is a stimulus? In J. Beck (Ed.), Organization and representation in perception. Hillsdale, $\mathrm{NJ}$ : Erlbaum.

JuLEsz, B. (1981). Figure and ground perception in briefly presented isodipol textures. In M. Kubovy \& J. R. Pomerantz (Eds.), Perceptual organization. Hillsdale, NJ: Erlbaum.

KoFFKA, K. (1935). Principles of Gestalt psychology. NY: Harcourt \& Brace.

MarR, D. (1982). Vision. San Francisco: Freeman.

PERKINS, D. N. (1972). Visual discrimination between rectangular and nonrectangular parallelopipeds. Perception \& Psychophysics, 12, 396-400.

Perkins, D. N., \& CoOper, R. G., JR. (1980). How the eye makes up what the light leaves out. In M. A. Hagan (Ed.), The perception of pictures (Vol. 2). New York: Academic Press.

Pomprantz, J. R. (1986). Visual form perception: An overview. In E. Schwab \& H. Nusbaum (Eds.), Pattern recognition by human and machines: Visual perception. New York: Academic Press.

Pomerantz, J. R., Schwattzere, S. D. (1975). Grouping by proximity: Selective attention measures. Perception \& Psychophysics, 18, 355-361.

Prinzmetal, W. (1981). Principles of feature integration in visual perception. Perception \& Psychophysics, 30, 330-340.

RAYNER, K. (1978). Foveal and parafoveal cues in reading. In J. Reguin (Ed.), Attention and performance VII (pp. 149-161). Hillsdale, NJ: Erlbaum.

Rock, I. (1983). The logic of perception. Cambridge, MA: A Bradford Book, MIT Press.

SHEPARD, R. N. (1981). Psychophysical complementarity. In M. Kubovy \& J. R. Pomerantz (Eds.), Perceptual organization. Hillsdale, NJ: Erlbaum.

Treisman, A., a Paterson, R. (1984). Emergent features, attention and object perception. Joumal of Experimental Psychology: Human Perception \& Performance, 10, 12-31.

Treisman, A., \& SoUTHER, J. (1985). Search asymmetry: A diagnostic for preattentive processing of separable features. Journal of Experimental Psychology: General, 114, 285-310.

von Gruenan, M. W., Wiggin, S., \& Reed, M. (1984). The local character of perspective organization. Perception \& Psychophysics, 35, 319-324.

(Manuscript received February 7, 1986; revision accepted for publication October $28,1986$. 